\title{
ANNUAL PRECIPITATION SERIES WAVELET ANALYSIS OF WELL-IRRIGATION AREA IN SANJIANG PLAIN
}

\author{
Dong Liu ${ }^{1}$, Qiang Fu, Yongsheng Ma ${ }^{1, *}$, Aihua Sun \\ ${ }^{1}$ School of Water Conservancy \& Civil Engineering, Northeast Agricultural University, \\ Harbin, Heilongjiang Province, P. R. China 150030 \\ * Corresponding author, Address: School of Water Conservancy \& Civil Engineering, \\ Northeast Agricultural University, Harbin 150030, Heilongjiang Province, P. R. China, \\ Tel: 13836137068, Fax:, Email: ysma66@126.com
}

\begin{abstract}
The rapid increase of paddy field acreage has led to widely descending of groundwater table in well irrigation paddy area of low-land in Sanjiang Plain recent years. More and more "hanging pump" and partial over pumping phenomenon have been occurring in well irrigation paddy area of low-land. Therefore, the groundwater resources in Sanjiang Plain need to be recharged urgently. For solving the mentioned problems upon, the authors took Farm 853 as an example, analyzed the law of multi-time scale series and characteristics of actual annual precipitation change in Farm 853 through using the wavelet theory, and the main periods of annual precipitation change and variation trend of drought-flood in this area were obviously. The study can provide scientific decision for fully utilizing precipitation, recovering groundwater and sustainable utilization in Farm 853 so much as the entire Sanjiang Plain.
\end{abstract}

Keywords: sanjiang plain, well irrigated paddy, annual precipitation time series, wavelet analysis, periods

\section{INTRODUCTION}

Sanjiang Plain lies in the eastern of Heilongjiang province; it is an alluvial plain of Heilongjiang, Songhua river and Wusuri river. The total area is 109 thousands $\mathrm{km}^{2}$, the area of cultivated land is 3667.7 thousands $\mathrm{hm}^{2}$ (Liang

Please use the following format when citing this chapter:

Liu, D., Fu, Q., Ma, Y. and Sun, A., 2009, in IFIP International Federation for Information Processing, Volume 293, Computer and Computing Technologies in Agriculture II, Volume 1, eds. D. Li, Z. Chunjiang, (Boston: Springer), pp. 563-572. 
Chunying et al., 2004), and it is an important commodity grain base of China. There is large area of lowland and flatland in Sanjiang plain. The soil of most area is heavy clay which the drainage capacity is low, and easyly leads to water logging disaster and yield reduction. From the 1980s, for reclaiming the lowland, many farms had begun to plant rice in Sanjiang plain, to control water logging and the action had obtained significant benefits. During 1981 to 2000 , the paddy acreage rapidly increased from 70 thousands $\mathrm{hm}^{2}$ to 953 thousands $\mathrm{hm}^{2}$ in Sanjiang plain and mainly is irrigated by well irrigation. The well irrigation paddy was 659 thousands $\mathrm{hm}^{2}$ in 2000. Owing to the paddy acreage rapidly increased and need to open large quantity of groundwater, the water consumption of well irrigation in Sanjiang plain was 4.56 billion $\mathrm{m}^{3}$ in 2000 . Additionally, the severe water wasting leads to groundwater table decrease rapidly. In Sanjiang plain, the 'hanging pump' and partial overdraft phenomenon often occur, the dynamic equilibrium of groundwater resources in Sanjiang plain has been severe damaged. Therefore, strengthen the study on groundwater recharge of Sanjiang plain is necessary. The precipitation is a main source and an important factor for groundwater artificial recharge. The multi-annual average precipitation is about $560 \mathrm{~mm}$, the annual variation of precipitation is great, and the annual distribution is very uneven (Li Shifeng et al., 2000). Therefore, study on the long term variation characteristics of annual precipitation in the area of lowland, well irrigation paddy, analysis the change law of drought and flood in Sanjiang plain are important for groundwater resources recovery and sustainable utilization.

The traditional methods that study the multiple time scale variation characteristics of precipitation mainly conclude Fourier analysis, Filtering analysis, Time series analysis etc, the study basis of these methods are only for single time scale, haven't the localization properties on both the time and frequency domains, and lack the mathematic rigorous on the diagnosis to the mutation points (Chen Huailiang et al., 2005), therefore they cannot comprehensively reflect the multiple time scale variation characteristics of precipitation. Wavelet analysis developed from 1980s, which has the time frequency multi-resolution function and strict mutation point analysis function in mathematic significance, it can simplify multiple time scale analysis (Chen Huailiang et al., 2005; Xu Yueqing et al., 2004), therefore better than all those traditional methods mentioned above and in recent years it has been widely used in the study on the climate multiple time scale analysis. The paper had taken the Farm 853 as a case, used the wavelet analysis theory to diagnose the multiple time scale characteristics of the 47 years precipitation time series of Farm 853, reveal the cycle characteristics of annual precipitation and change trend of drought and flood in the Farm 853 , provided the scientific basis for the groundwater recovery and key problems study on regional water saving agriculture. 


\section{THE BASIC PRINCIPLE OF WAVELET ANALYSIS}

\subsection{Wavelet function}

There are many kinds of wavelet function, often using Marr wavelet, Morlet wavelet and Wave wavelet etc. in the wavelet analysis (Ou Suying et al., 2004; Liu Zhongyang et al., 2005; I. I. I ppolitov et al., 2002; Wang Wensheng et al., 2005). This paper selected the Morlet wavelet that is better in both the time and frequency domain locality, the function is

$$
\psi(t)=e^{i c t} e^{-t^{2} / 2}
$$

Where: $c_{\text {is constant, }} i$ is imaginary number.

Because Morlet wavelet is obtained by a periodic function though Gaussian smoothing, there is corresponding relationship between its scale factors and the period in Fourier transform: $T=\left[4 \pi /\left(c+\sqrt{2+c^{2}}\right)\right] \times a$. When take the constant $c=6.2, T=1.00057 a \approx a$, therefore the Morlet can be used to periodic analysis.

\subsection{Wavelet transformation}

Wavelet transformation is the key of the Wavelet analysis. For the Wavelet function $\psi(t)$ given and satisfy some conditions, the constant Wavelet transformation of time series $f(t) \in L^{2}(R)$ defined as:

$$
W_{f}(a, b)=|a|^{-\frac{1}{2}} \int_{-\infty}^{+\infty} f(t) \bar{\psi}\left(\frac{t-b}{a}\right) d t
$$

Where: $W_{f}(a, b)$ is Wavelet transformation coefficient, $a$ is scale factor, as the length of Wavelet period, and $b$ is time factor, as the translation time. (Liu Zhongyang et al., 2005; Wang Wensheng et al., 2005; Wu Dongjie et al., 2004)

The hydrological time series are mostly discrete in practical application, for example $f(k \Delta t) \quad(k=1,2, \cdots, n ; \Delta t$ is sampling time interval). The formula (2) can be revised (Liu Zhongyang et al., 2005; I. I. Ippolitov et al., 2002; A. Subasi et al., 2005) as:

$$
W_{f}(a, b)=|a|^{-\frac{1}{2}} \Delta t \sum_{k=1}^{n} f(k \Delta t) \bar{\psi}\left(\frac{k \Delta t-b}{a}\right)
$$


Where: $W_{f}(a, b)$ is a function of time domain parameter and frequency domain parameter, it is the output of filter that the time series of $f(t)$ or $f(k \Delta t)$ response by unit pulse. When $a$ is smaller, the resolution of frequency domain is lower, and the resolution of time domain is higher; when $a$ is bigger, the resolution of frequency domain is higher, and the resolution of time domain is lower. Therefore, the Wavelet transformation can realize time and frequency localization that window size fixed and shape variable.

According to $W_{f}(a, b)$ changed by $a$ and $b$, we can draw the two dimensional contour map about $W_{f}(a, b)$, with $b$ as abscissa and $a$ as ordinate, called the Wavelet transformation parameter map. With analysis of Wavelet transformation parameter map, we can obtain the Wavelet change characteristics that hydrological time series in Wavelet change domain, and reveal the multiple time scale evolution characteristics and mutation characteristics of the hydrological time series.

\subsection{Wavelet variance}

Wavelet variance is the integration of the square of all the Wavelet transformation parameter about $a$ in the time domain. For the discrete hydrology time series, Wavelet variance can be calculated with the formula (4) (X. C. Mi et al., 2005; Liu Jianmei et al., 2005)

$$
\operatorname{Var}(a)=\frac{1}{n} \sum_{b=1}^{n}\left|W_{f}(a, b)\right|^{2}
$$

Where: $n$ is the number of samples, $\left|W_{f}(a, b)\right|^{2}$ is module square of Wavelet transformation coefficient.

The wavelet variance change process follows the scale $a$ is called wavelet variance graph, the graph can show the fluctuation of all kinds of scales including hydrological time series and the characteristic of its intensity (the size of energy) follows the scale's change, in the graph each peak value respectively corresponds to each significantly period. Therefore, we can conveniently determine a main time scale (main period) existing in a time series.

\section{THE CASE STUDY}

The Farm 853 located in the eastern of Sanjiang plain of Heilongjiang province, belonging to Hongxinglong farm management bureau, the total 
area of the land is $1168.7 \mathrm{~km}^{2}$; the area of cultivated land among them is $581.35 \mathrm{~km}^{2}$. The Farm 853 is a large mechanized state farm which mainly plants wheat, soybean, maize and rice. From 1991 to 2000, the paddy field acreage of the Farm 853 rapidly increases from 6.1 thousand $\mathrm{hm}^{2}$ to 26.7 thousand $\mathrm{hm}^{2}$ which $72 \%$ of paddy is well irrigation rice. According to investigation data of the Farm 853 in the 8 years from 1991 to 1998, the groundwater table descends $0.3 \mathrm{~m} / \mathrm{a}$. The annual average exploitable capacity of groundwater resources is 0.04186 billion $\mathrm{m}^{3} / \mathrm{a}$. However, the amount of exploitation of groundwater resources in 2000 is 0.08046 billion $\mathrm{m}^{3}$, the annual average amplitude of groundwater table descent is $0.3 \mathrm{~m} / \mathrm{a}$ in recent 6 years from 1999 to 2004. The continuous decrease of groundwater level has severe damaged the balance of local groundwater resources. In order to save the groundwater resources and realize the sustainable development of groundwater resources, this paper set the Farm 853 as an example, according to the wavelet analysis theory mentioned above, the multiple time scale analysis for measured series of annual precipitation data in Farm 853 has been analyzed and know the detail structure and change trend in different time scale.

\subsection{Data collection and treatment}

The data of annual precipitation from 1959 to $2005(n=47)$ have been collected from the meteorological observatory in the Farm 853. For treatment convenient, the measured sequence data of annual precipitation was anomaly treated (that is centralization), and drew the curve of annual precipitation series, see figure 1 .

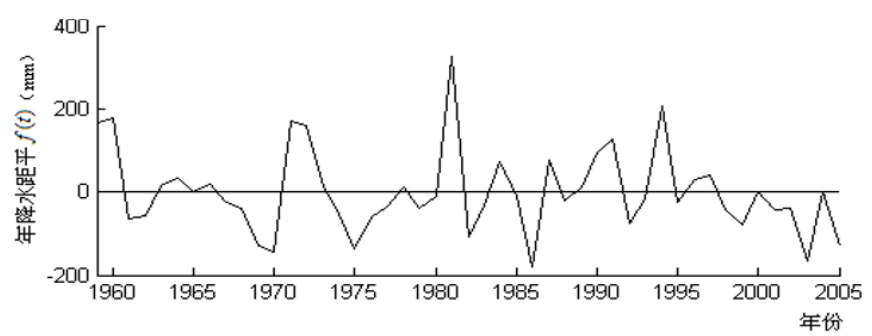

Fig. 1 The annual precipitation curve of Farm 853 from1959 to 2005 


\subsection{Wavelet transformation of annual precipitation anomaly sequence}

According to the method mentioned above, the wavelet transformation coefficient $W_{f}(a, b)$ of annual precipitation anomaly sequence $f(k \Delta t)$ ( $k=1,2, \cdots, 47 ; \Delta t=1$ ) in the Farm 853 has been calculated.

Because the Morlet wavelet belongs to complex wavelet, its wavelet transformation coefficient concludes the real part and deficiency part. Learn from the literature (X. C. Mi et al., 2005, Zhang Shaowen et al., 2004; Cui Fenglin et al., 2000), the module square of wavelet transformation is proportional to the energy that the function $f(t)$ in its wavelet transformation domain, $\left|W_{f}(a, b)\right|^{2}$ is wavelet spectrum energy when signal is at $a, b$, therefore the module square of wavelet transformation can be used to represent the energy intensity of the characteristic time scale signals. However, the message of the distribution and phase two aspects that different characteristics time scale signals showed in different time can be represented by the real part of wavelet transformation. Therefore, the module square and real apart of wavelet transformation coefficient $W_{f}(a, b)$ of annual precipitation anomaly sequence implicates the characteristics messages that the sequence changed by the time scale $a_{\text {(namely periodic } T \text { ) }}$ and time shift.

\subsection{Time and frequency analysis of annual precipitation anomaly sequence}

According to the methods mentioned above, drawing the module square (see figure 2) and real apart (see figure3) contour maps of the wavelet transformation coefficient of the Farm 853 annual precipitation anomaly sequence, and analyze the time and frequency change of annual precipitation anomaly sequence.

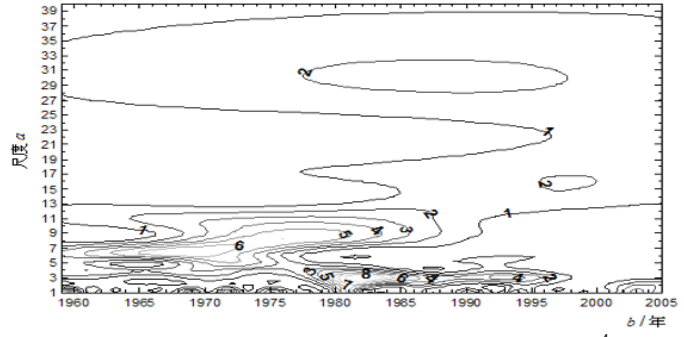

Fig.2:The wavelet transform coefficients modulus square $\left(\times 10^{4}\right)$ isoline of annual precipitation anomaly series of Farm 853 


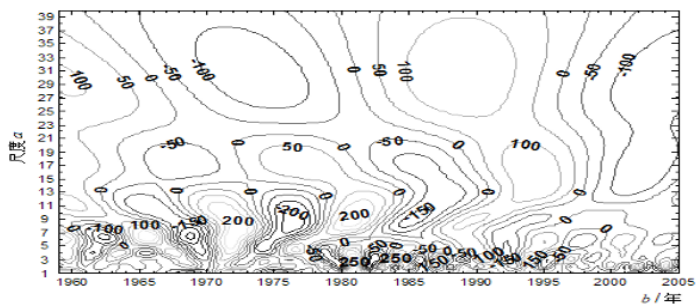

Fig.3: The wavelet transform coefficients real part time-frequency distribution of annual precipitation anomaly series of Farm 853

\subsubsection{The time and frequency analysis of wavelet transformation coefficient module square}

The intensity of energy distribution of different time scale signal can be seen as Figure 2, in which the energy change of $2 \sim 4$ years time scale signal is the strongest, occurred from 1978 to 1996 . The oscillation center is about 1981; the energy change of $4 \sim 12$ years time scale signal is stronger, mainly occurred from 1959 to 1988 , the oscillation center is near by 1971 ; the 25 37 years time scale signal mainly occurred from 1959 2000; the $1 \sim 2$ years time scale signal mainly occurred from 1959 1961, 1968 1973, 1974 1977, 1982 1985 and 1987 1993; the energy change of the other stages time scale signal are weaker.

\subsubsection{The time and frequency analysis of wavelet transformation coefficient real part}

The change of different time scale, the distribution of catastrophe points and its phase structure as shown in the Figure 3, the time scale of $1 \sim 4$ years, $4 \sim 12$ years and $25 \sim 37$ years represent the most obvious, positive and negative phase appeared alternative, its central time scales are about 3 years, 7 years and 30 years respectively. In addition, the $12 \sim 21$ years time scale also has representation, its central time scale is about 17 years. In order to show the fluctuation characteristics that drought and flood of the Farm 853 annual precipitation anomaly sequence changed alternative, fixed the value of the time scale $a$ (respectively $=3,7,17,30$ ), made a cutting line paralleled with the axis $b$, and took a point on the cutting line, made the hydrograph that the real part (expressed as $R\left[W_{f}(a, b)\right]$ ) of wavelet transformation coefficient $W_{f}(a, b)$ changed with the time shift $b$, see Figure 4. 


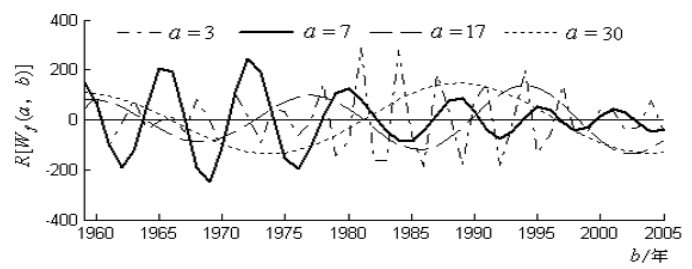

Fig.4: The Morlet wavelet transform real part variation course of annual precipitation anomaly series

The figure 4 shows that the real part change process of wavelet transformation coefficient of different time scale, now take the 7 years time scale for an example, analyze the drought and flood change of precipitation and the location of the transformation points of positive and negative phrase. According to the real part change process of wavelet transformation coefficient of different time scale in the figure 4, obtained a conclusion that before 1960, 1964 1967, 1971 1974, 1978 1982, 1987 1990, 1994 1996, 2000 2002 are positive phase, which express the abnormal large amount of rain; from 1961 1963, 1968 1970, 1975 1977, 1983 1986, 1991 1993, 1997 1999 and after 2003 are negative phase, which express the abnormal small amount of rain; 1961, 1964, 1967, 1970, 1974, 1978, 1982, 1987, 1990, 1994, 1997, 2000 and 2003 are the catastrophe point of drought and flood.

\subsection{Main period analysis of annual precipitation sequence}

The main period of the annual precipitation sequence of Farm 853 changed with the time can be studied by the wavelet variance. According to the methods mentioned above, using the wavelet transformation coefficient has solved in different scales, and by means of computer programming, draw the wavelet variance of annual precipitation anomaly sequence, see figure 5 . Seeing from the figure 5, the main peaks of wavelet variance respectively occurred at the point which the scale $a$ equals to 3, 7, 17 and 30, the first peak is the wavelet variance which the scale $a=3$ corresponds to, shows that the period about 3 years oscillated strongest, it is the first main period, the second, the third and the forth main period is 7 years, 30 years and 17 years respectively.

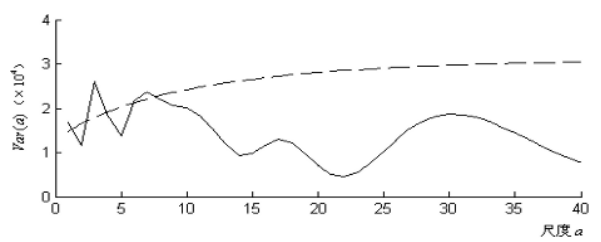

Fig.5: The wavelet variance of annual precipitation anomaly series in Farm 853 (solid line is wavelet variance, dashed line is $95 \%$ confidence level) 


\subsection{The trend analysis of annual precipitation sequence change}

Figure. 4 has given the real part change process of the wavelet transformation coefficient in each main period, the change trend of precipitation of the Farm 853 in different time scale can be analyzed by means of the figure 4 . From the trend analysis of the change of smaller scale 3 years and 7 years and the bigger scale 17 years. It shows that from 2006 to 2007 the precipitation of the Farm 853 was on the less side, after 2008 is the stage that precipitation is on the high side; from the trend analysis of the change of 30 years scale, we can see that from 2006 to 2011 the precipitation of the Farm 853 is on the less stage, however in the 15 years after 2012, the precipitation of the Farm 853 is on the high stage.

\section{CONCLUSION}

Wavelet analysis can unfold the fine structure of the hydrological time sequence, and offer a new way for analysis the change characteristics and mutation characteristics of the multiple time scale of the hydrological time sequence.

The annual precipitation sequence of the Farm 853 may has 3 years, 7 years, 17 years and 30 years altogether four main periods was recognized by wavelet analysis, in the four main periods only the 3 years and 7 years main period are obviously and the main periods of 17 years and 30 years has not been clearly.

Through wavelet analysis, the change trend of precipitation of the Farm 853 was roughly opened out, we can use fully the rain and flood resources in the stage of precipitation on the high side to artificial recharge groundwater, speed up the recovery of groundwater resources, thus realize the sustainable utilization of local groundwater resources.

\section{ACKNOWLEDGEMENTS}

Funding for this research was provided by National Natural Science Foundation (No.30400275); Northeast Agricultural University innovative project (No. CXZ009). 


\section{REFERENCES}

A. Subasi, M. K. Kiymik, M. Akin et al. Automatic recognition of vigilance state by using a wavelet-based artificial neural network [J].Neural Comput \& Applic, 2005, 14 (1) : $45-55$.

Chen Huailiang, Hu Peng, Zhang Xufeng etc. Multiple Time Scales Analysis on Agro-climate Taking Wheat and Maize in Zhengzhou City as an Example[J]. Journal of Natural Resources,2005,20(6):814-821.

Cui Fenglin, Xie Chunlai, Fu Lei, et al. The Time Frequency Spectrum of Wavelet Transform and Its Application in Seismic Sequence Identification [J]. Journal of Changchun University of Science and Technology, 2000,30(4):397-399.

I. I. Ippolitov, M. V. Kabanov, S. V. Loginov. Wavelet Analysis of Hidden Periodicities in Some Indexes of Solar Activity[J].Russian Physics Journal, 2002， 45 (11):1086-1092.

Li Shifeng, Cui Guangchen, Yang Guoshun. Flood Disasters and Treatment Measures in Sanjiang Plain [J]. Advances in Science and Technology of Water Resources,2000,20(1):65 $-67$.

Liang Chunying, Wang Xi, Yang Tianxiong. Precision Agricultures Application Research and Discussion in Sanjiang Plain[J]. Journal of Agricultural Mechanization Research,2004,(3):193-194.

Liu Jianmei, Wang Anzhi, Pei Tiefan, et al. Wavelet Analysis on the Runoff Trend and Periodic Change Characteristics of Zagunao River [J]. Journal of Beijing Forestry University, 2005,27(4):49-55.

Liu Zhongyang, Wang Yong, Ding Yuanyuan et al. Multiple Time-Scale Analysis of Precipitation Variation in Zhengzhou during Last 54 Years [J]. Meteorological Science, 2005, 33(suppl.):123-126.

Ou Suying, Chen Zisang. Application of Wavelet Transformation to Relative Sea Level Research [J]. Geographical Science, 2004,24(3):358-364.

Wang Shaohua, Tian Yuan. Preliminary Research on Groundwater Table Change and Causes in Sanjiang Plain[J].Journal of Irrigation and Drainage, 2003,22(2):61-64.

Wang Wensheng, Ding Jing, Li Yueqing. Wavelet Analysis on Hydrology[M].Beijing: Journal of Chemical Industry, 2005, 115-141.

Wu Dongjie, Wang Jinsheng, Teng Yanguo. Prediction the Dynamic Groundwater Level with Wavelet Decomposition and Transform Method [J]. Journal of Hydraulic Engineering,2004,(5):39-45.

X. C. Mi, H. B. Ren, Z. S. Ouyang et al. The Use of The Mexican Hat and The Morlet Wavelets for Detection of Ecological Patterns[J].Plant Ecology, 2005, 179 (1) : 119.

$\mathrm{Xu}$ Yueqing, Li Shuangcheng, Cai Yunlong. Study on the Precipitation Change Rule of Heibei Plain Based on Wavelet Analysis [J]. Science in China, Ser.D, 2004,34(12):11761183.

Zhang Shaowen, Ding Jing, LIAO Jie, et al. Analysis of Natural Annual flow Time Series in the Upper Reach of the Yellow River Based on Wavelet Transform [J]. Journal of Sichuan University (Engineering Science Edition), 2004, 36(3):32-37. 